\title{
Design of Individual Brace for the 3-D Correction and Mechanics Study
}

\author{
Wenzhong $\mathrm{Nie}^{1, \mathrm{a}}$, Guangkai Fang ${ }^{1, \mathrm{~b}}$ and Yongzeng Yan $^{1, \mathrm{c}}$ \\ ${ }^{1}$ School of Mechanical Engineering, Shanghai Institute of Technology, Shanghai 201418, China. \\ aniewz@163.com, bfangguangkai@163.com, cyanyongzeng2007@126.com
}

Keywords: IS, Finite Element, Individual Brace, Mechanics.

\begin{abstract}
Establish 3-D geometry model including thoracic-lumbar-sacrum, thorax and pelvis based on CT data in IS patient, and then construct a completely finite element model based on the human anatomy. On the basis research on rapid design method of individual brace, simulate clinical spine stress by reasonably setting gaskets distribution, analysis the effect of different orthopedic force and position on orthopedic effect and assessment, conduct mechanics analysis with orthopedic effect according to the clinical practice to determine the optimal brace design scheme.
\end{abstract}

\section{Introduction}

For patients with Cobb is below 40 degrees, brace treatment is currently the only effective non-surgical treatment [1]. Therefore, the study of pathogenesis of IS and orthopedic brace scheme has great real sense.

Existing brace treatment for the correction effects is mainly concentrated in the spine of coronal, sagittal and transverse synchronous rectification is not obvious, so 3-D correction cannot be achieved. Meanwhile there is poor compliance, side effects like flat-back and problem such as unable to personalize.This study attempts to design a new individual brace that fully reflects the patients' individual biomechanical characteristics and achieves the 3-D correction. Finally, through the simulation analysis validates orthopedic effect. So as to improve the correction and avoidance of surgery for high health expenditure, reduce the burden on families and society; produce good economic and social benefits.

\section{Specific Process}

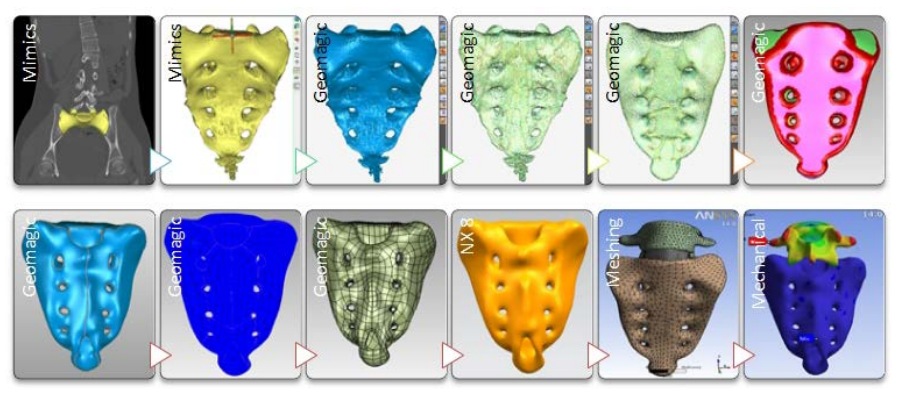

Fig. 1 To the sacrum as an example to display the FEA process

Build Trunk Structure of 3-D Geometric Model. Equipment: TOSHIBA/Aquilion ONE 320-row, parameters:120KV 200.00mAs.Refer to knowledge of human anatomy, use Mimics Innovation Suite 15, Geomagic Studio 2014 and NX 8, build a fully trunk 3-D geometric model includes the thoracic-lumbar-sacral, thorax and pelvis, lay the foundation for further study.

Build Finite Element Model. Integrated use of HyperMesh 12 and ANSYS 15, choose element type, material properties and contact type. Among them, set the inner surface of brace and the outer surface of the human body model for surface-surface contact, complete the mesh to establish finite element model [2].

Finite Element Model Validation. On the basis of geometric similarity validation (as Fig. 2), refer to the relevant literature [3], using authoritative mechanical study results to verify the validity of the section (as Table 1 and Table 2), so proved that the model is valid. 


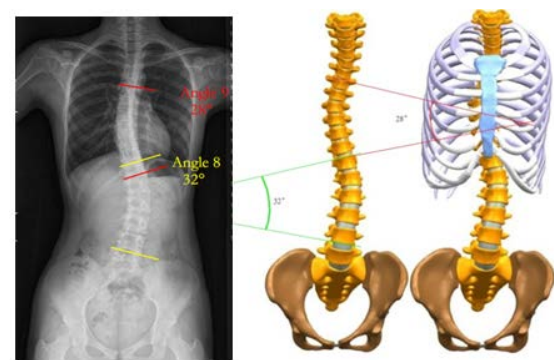

Fig. 2 Compare measured Cobb of bend in the LAT on X-ray and model Table 1 Comparison of ROM of L4-L5 in the 8Nm with Xu and Chan

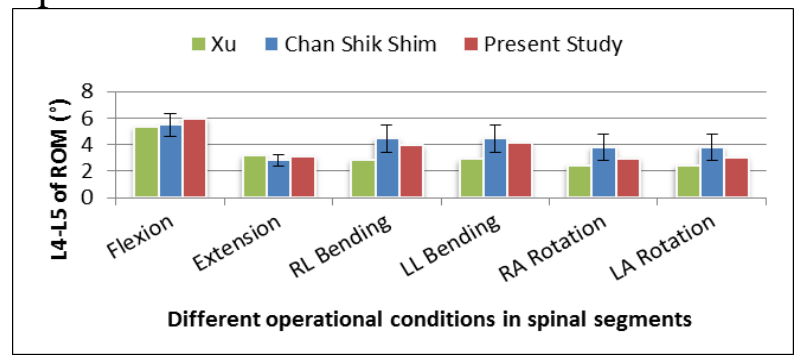

Table 2 Comparison of ROM of L5-S in the 10Nm with Wang and Isao

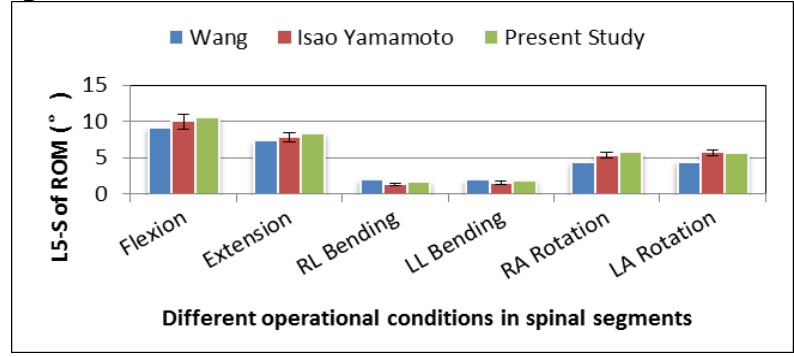

Modeling of Individual Brace and Abdominal Wall. Modeling process: As shown in Fig. 3(1, Sagittal section borders; 2, Extract the raster image in the NX; 3, Fully brace model), adjust frontal view of the spine model in Mimics and NX. First of all as the upper boundary at the specified rib A and as a lower boundary at the pelvis $\mathrm{D}$, insert a cross-sectional view of the corresponding location in Mimics in the form of raster images to the new datum plane of the model in the same location in the NX by measuring tools. Select the curve profile as contours of the upper and lower boundary. Similarly, select 8 cross sections from section A to section D in lateral view, then, get 10 contours in the NX. Through the surface command, use 10 profile curves to create a complete surface. Generate original geometric model of individual brace along the curved outer thickening-oriented $5 \mathrm{~mm}$ and optimized for easy wearing. According to the characteristic of deformity by using Insert the Conventional Pad to design gasket in different locations. Abdominal model the same as above. Fully model reflects the patient's individual mechanics.
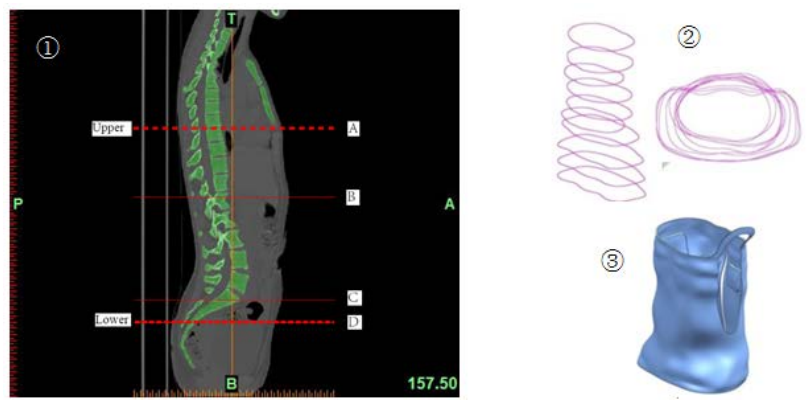

Fig. 3 Modeling of abdominal wall and brace

The Effect of the Brace Correction in Simulated Clinical.Refer to related literature, set spine loading boundary conditions as follows [3, 7]: Constraint T1 freedom in the YZ plane and ilium all freedom; Put combined force FY and FX on the YZ and ZX planes, Load G1: FX=0, FY=60N; G2: $F X=20 N, F Y=40 N ; G 3: F X=30 N, F Y=30 N ; G 4: F X=40 N, F Y=20 N ; G 5: F X=60 N, F Y=0$. The 
measured angle from X-ray: Thoracic $\mathrm{Cobb}=28^{\circ}$, Lumbar $\mathrm{Cobb}=32^{\circ}$, Thoracic kyphosis $=15^{\circ}$, Lumbar lordosis $=43^{\circ}$, $\mathrm{T} 8$ rotation $=10^{\circ}$, L2 rotation $=19^{\circ}$.

Table 3 Comparison of scoliosis load before and after

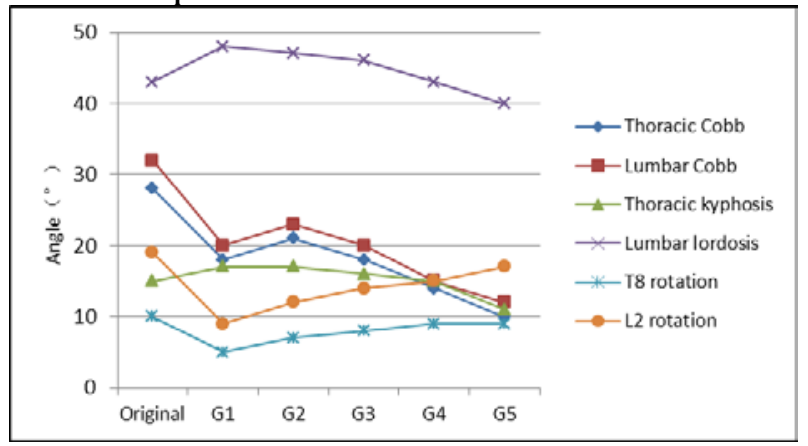

For models that overlay well, as shown in Fig. 4, load separately 20, 40 and 60N tightening force on the brace corresponding to the Thoracic, Lumbar and Pelvis in three parts, boundary conditions as above, through the gaskets transfer combined loads.

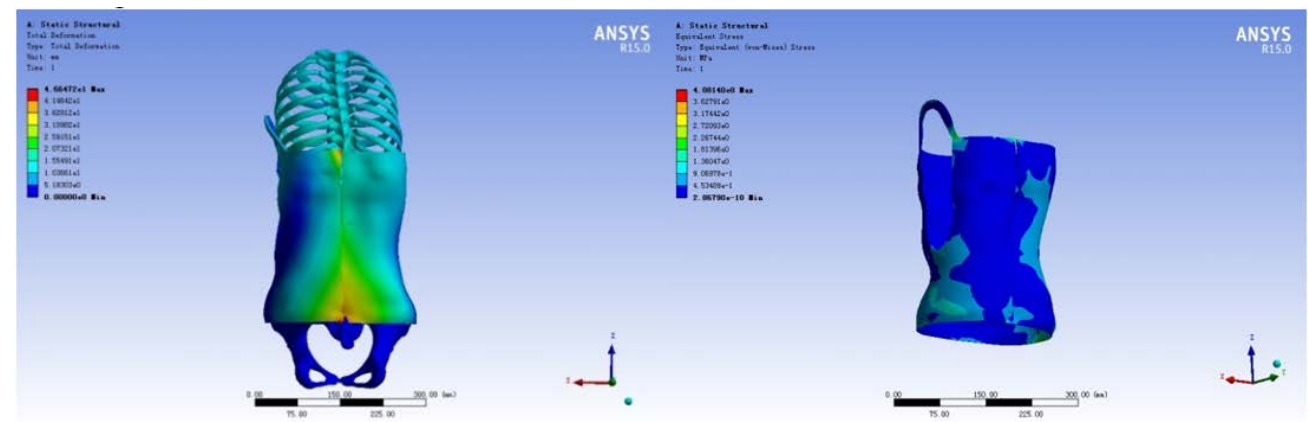

Fig. 4 Superposed loads position and deformation and 60N force brace stress contour

\section{Discussion}

In a simulated clinical spine stress scenarios, Table 3 shows that with increasing force FX, thoracolumbar Cobb angle decreases, scoliosis correction rates increased; Thoracic kyphosis and Lumbar lordosis angle becomes small, but not be obvious; with increasing force FY, scoliosis spinal vertebral sagittal rotation angle reduce, combined force of comprehensive orthopedic effect is good. Found in the simulation, when tightening force exerted on the brace, the stress of three parts obvious changes, show that individual correction brace is sensitive to tightening force, through cloud observed, in other parts of the model is not obvious, means individual brace for normal parts will not cause discomfort. The stress is larger in thoracic and lumbar convex, Maximum stress occurs in L2, stress values for the 5.92Mpa. Convex side and the rear of the vertebral body stress greater than the concave side, higher stress levels than the cancellous bone of the cortical bone, maximum pressure values appear on L2-L3 segment of the convex side. The disc of the largest stress is located in anomalies of the convex side of the spine, between maximum stress values are located in the L2-L3 section, and stress value is $2.96 \mathrm{Mpa}$. When applying the $60 \mathrm{~N}$ tightening force, the correction rate can reach more than $60 \%$; with the imposed on the sagittal spinal orthopedic force increases, coronal correction improved. Meanwhile, vertebral orthopedic effect of rotation is more obvious. According to the characteristic of scoliosis, set gaskets to provide lordosis correction force on the right Thoracic and left Pelvis, and the back of T-L segment. Compare to pure simulation spinal deformity correction, for the coronal and sagittal planes with better orthopedic effect, as shown in Table 4.

Individual brace for the 3-D correction makes up for the deficiency of Boston and Milwaukee brace to axial rotation correction and doesn't produce side effects such as flat-back. Overall effects better than previous literature reported. When tightening force of $60 \mathrm{~N}$ brace stress contour indicate that gaskets had played a full correction (as Fig.4). Brace design is reasonable and effective. 
Table 4 The change of spine curve on loading different tightening force

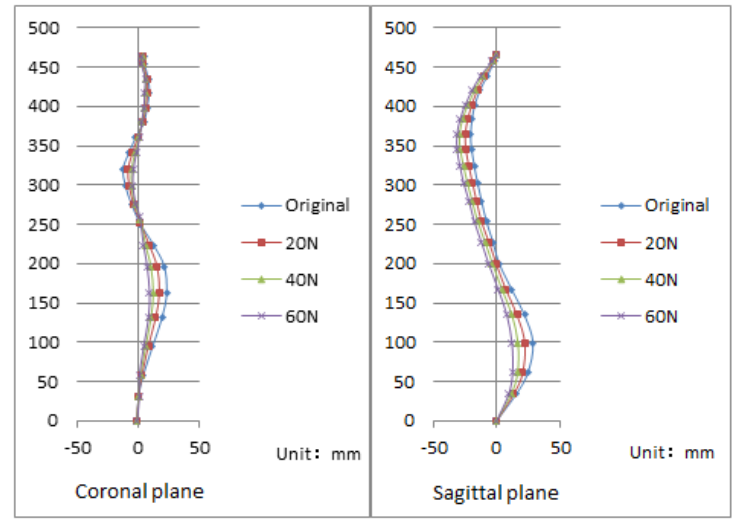

Therefore, the proposed research method provides a new direction for conservative treatment, make up for the shortcomings of the traditional brace. Fully conform to the curve of patients with spinal deformity and setting up reasonable individual gaskets location can truly realize the 3-D correction of scoliosis. This method can improve the researchers to design and optimize personalized correction efficiency of brace and brace treatment effects, avoid surgery, reduce the burden on patients, has good meaning and application.

\section{Conclusions}

For the establishment of 3-D finite element model of IS provides an easy and accurate method, particularly on the basis of this model, innovatively present an individual correction brace with rapid design courses. By simulating the clinical effect of spinal and orthopedic, proved that the brace is an effective and reliable. But the actual effect for correction brace still requires further clinical tests.

\section{References}

[1] Parent S, Newton P O, Wenger D R. Adolescent idiopathic scoliosis: etiology, anatomy, natural history, and bracing [J]. Instructional course lectures, 2004, 54: 529-536.

[2] Goel V K, Monroe B T, Gilbertson L G, et al. Interlaminar Shear Stresses and Laminae Separation in a Disc: Finite Element Analysis of the L3-L4 Motion Segment Subjected to Axial Compressive Loads[J]. Spine, 1995, 20(6): 689-698.

[3] Clin J, Aubin C E, Parent S, et al. Comparison of the biomechanical 3D efficiency of different brace designs for the treatment of scoliosis using a finite element model [J]. European Spine Journal, 2010, 19(7): 1169-1178.

[4] Van Loon P J M, Kühbauch B A G, Thunnissen F B. Forced lordosis on the thoracolumbar junction can correct coronal plane deformity in adolescents with double major curve pattern idiopathic scoliosis[J]. Spine, 2008, 33(7): 797-801.

[5] Ni H, Zhu X, He S, et al. An increased kyphosis of the thoracolumbar junction is correlated to more axial vertebral rotation in thoracolumbar/lumbar adolescent idiopathic scoliosis [J]. Spine, 2010, 35(23): 1334-1338.

[6] Perie D, Aubin C E, Lacroix M, et al. Biomechanical modelling of orthotic treatment of the scoliotic spine including a detailed representation of the brace-torso interface [J]. Medical and Biological Engineering and Computing, 2004, 42(3): 339-344.

[7] Cheng F H, Shih S L, Chou W K, et al. Finite element analysis of the scoliotic spine under different loading conditions[J]. Bio-medical materials and engineering, 2010, 20(5): 251-259. 